\title{
La discapacidad como factor de vulneración del derecho a la salud y rehabilitación en mujeres privadas de libertad
}

\section{Disability as a vulnerability factor of the right to health and rehabilitation in women deprived of freedom}

\author{
Evelyn Vera Alfonso ${ }^{a}$
}

\begin{abstract}
Resumen
Numerosas personas con discapacidad se encuentran privadas de su libertad, por ser sospechosas o culpables de hechos punibles y el Estado es el encargado de garantizar que todos los derechos fundamentales se cumplan aun estando en las instalaciones de una penitenciaría. El presente estudio se propuso analizar las condiciones de salud de mujeres con discapacidad privadas de libertad desde la percepción de actores clave de un Penal, en el marco del enfoque de derechos. Fue un estudio exploratorio, con enfoque cualitativo. Realizado en el marco del Trabajo Práctico de la Asignatura Investigación Social y Trabajo Social III del Instituto de Trabajo Social de la UNA, con el apoyo del Mecanismo de Prevención contra la Tortura. La técnica utilizada fue la entrevista en profundidad. Como instrumento se utilizó una guía de preguntas que contenía las categorías de análisis a ser estudiadas. La muestra estuvo constituida por profesionales de las áreas de interés ubicadas dentro del penal, como ser Trabajo Social, Psicología y Judiciales. Se resguardó la identidad de las personas entrevistadas, así como las denuncias y/o situaciones que fueron comentadas durante las entrevistas por mujeres privadas de su libertad, o por funcionarios del penal. Se pudo concluir que, al momento del levantamiento de los datos, el Penal no contaba con un protocolo de atención o ruta de acción para mujeres con discapacidad que son privadas de su libertad. Lo que trae como consecuencia que las mismas vivan en una situación de constante vulneración de derechos y a lo que le sumamos el factor agravante de que no acceden a un servicio de atención o rehabilitación aun cuando lo necesitan por su condición.
\end{abstract}

Palabras clave: discapaidad, mujeres privadas de libertad.

\footnotetext{
Abstract

Many people with disabilities are deprived of their liberty, for being suspects or guilty of punishable acts, and the State is in charge of guaranteeing that all fundamental rights are fulfilled even in the
}

Kera Yvoty: reflexiones sobre la cuestión social. Vol. 2, 2017, 117-122.

ISSN (impreso): 2519-7797

a Universidad Nacional de Asunción, Instituto de Trabajo Social, Paraguay.

Correspondencia a: evelynverao7@hotmail.com

Cita:

Vera Alfonso, E. (2017). La discapacidad como factor de vulneración del derecho a la salud y rehabilitación en mujeres privadas de libertad. Kera Yvoty: reflexiones sobre la cuestión social, 2, 117-122.

Recibido:

7 octubre 2017

Aceptado:

27 noviembre 2017 
penitentiary facilities. The present study aimed to analyze the conditions of women with disabilities deprived of freedom's health, from the perception of key actors of a prison, within the framework of rights approach. It was an exploratory study, with a qualitative approach, performed in the framework of Practical Work from the subject "Social Investigation and Social Work III" of the Social Work Institute of the UNA, with the support of the Mechanism of Prevention Against Torture. The technique used was in-depth interviews. A question guide containing the categories of analysis to be studied was used as an instrument. The sample consisted of professionals from the areas of interest, located within the prison, such as Social Work, Psychology and Judicial. The identity of people interviewed was safeguarded, as well as the complaints and/or situations that were commented during the interviews, by women deprived of the liberty, or by correctional officers. The conclusion at the moment of data collection was that the prison did not have a service protocol or route of action for women with disabilities deprived of their liberty. As a consequence they live in a situation of constant infringment of rights, to which we add the aggravating factor they do not access a care of rehabilitation service, even when they need it due to their condition.

Keywords: disability, freedom deprivation women.

\section{Introducción}

En Paraguay, históricamente las personas con discapacidad fueron conceptualizadas como objetos, la sociedad a través de la medicina y los pre-conceptos creados por la mayoría de las personas y los profesionales, entendían a la discapacidad como un problema, como algo que debía de corregirse, como una carga para la familia y la comunidad. Se encontraban invisibilizadas, escondidas y condenadas al no acceso a ningún derecho por el hecho de no ser "normal" (Moscoso, 2011). La normalidad era entendida desde el punto de que cada uno de nosotros, como ciudadanos de tenemos características únicas y comunes, donde aquel que no cumpla o se salga de las mismas debe ser tratado y apartado hasta que pueda volver a reinsertarse (Brogna, 2006). Desde el año 2008, año en que entra en vigor la Convención Internacional por los Derechos de las Personas con Discapacidad (CIDPD), la sociedad ha evolucionado a reconocer a las mismas como "personas" y sujetos de derechos.

El propósito de la Convención (CIDPD, 2008) en su artículo 1 fue: promover, proteger y asegurar el goce pleno y en condiciones de igualdad de todos los derechos humanos y libertades fundamentales por todas las personas con discapacidad, y promover el respeto de su dignidad inherente. Las personas con discapacidad incluyen a aquellas que tengan deficiencias físicas, mentales, intelectuales o sensoriales a largo plazo que, al interactuar con diversas barreras, puedan impedir su participación plena y efectiva en la sociedad, en igualdad de condiciones con las demás.

En la actualidad, muchas personas con discapacidad se encuentran privadas de su libertad, por ser sospechosas o culpables de hechos punibles y el Estado es el encargado de garantizar que todos los derechos fundamentales se cumplan aun estando en las instalaciones de una penitenciaría (CEJIL, 2006). Sin embargo, pocos son los estudios, investigaciones o informes, que develan la situación real que viven las personas con discapacidad privadas de su libertad, y siguen siendo invisibilizadas en un contexto que de por sí, es un factor que aumenta la posibilidad de vulneración de derechos humanos (MNP, 2014).

En el caso de las mujeres con discapacidad privadas de libertad, se le suman una serie de factores que profundizan la discriminación y exclusión. Es por eso que a través de esta investigación se pretendió analizar las condiciones de 
salud de las mujeres con discapacidad privadas de su libertad en el Penal del Buen Pastor, con un enfoque de derechos humanos (Bareiro, 2003).

En general, el único derecho que el juez o jueza priva a una persona que entra a la cárcel por orden judicial es el de la libertad ambulatoria. Sin embargo, en nuestro país, son varios los derechos que en la práctica no son cumplidos para las internas y se ha señalado que la situación es grave y va deteriorándose (MNP, 2015).

Un breve resumen de las condiciones que presentan las personas privadas de libertad en las cárceles de Paraguay, habla de que las mismas están sobrepobladas, con denuncias sobre realidades alarmantes durante todo el 2014. Los datos refieren que la mayoría de las personas privadas de libertad, aproximadamente un $75 \%$, se encuentra en prisión preventiva, con lo cual se asume de antemano que son culpables y no existe la presunción de su inocencia. (MNP, 2014).

Además de la sobrepoblación y sus implicancias, otro aspecto analizado en el Informe de MNP del año 2014 es lo relativo a los tipos de hechos punibles por los cuales son privadas de libertad las mujeres, ya que el $63 \%$ ingresó por una causa vinculada a la Ley nro. 1340, que reprime el tráfico ilícito de drogas. En la casa del "Buen Pastor" es donde más se nota este perfil de internas. (MNP, 2014).

Las mujeres con discapacidad en privación de libertad, además de las condicionantes propias de dicha privación (encierro, hacinamiento, problemas de relacionamiento, alimentación y salud que no son apropiadas para un ambiente que favorezca a la salud mental), están expuestas a situaciones de vulneración y sufren otras condicionantes debido a su condición psicofísica por una inadecuada infraestructura, ausencia de servicios sanitarios especializados, de programas de rehabilitación, entre otros

Existe un conjunto de instrumentos, algunos basados en legislaciones nacionales y convenios internacionales para adoptar medidas que tiendan a la igualdad y no discriminación (SENADIS, 2014).

Toda persona tiene el derecho, la capacidad y la libertad de dirigir su vida, de acceder a la educación, a la salud y a un empleo en igual condiciones, pero en nuestra sociedad las personas con discapacidad se toman con las barreras físicas, las actitudinales y las comunicacionales quedando obligados a ser personas que no ejercen derechos de forma independiente, que no acceden a una vida "normal" como todas las demás.

Todas esas barreras construidas por una sociedad cerrada y por un sistema hegemónico que excluye, segrega a varios grupos determinados, entre ellos a las personas con discapacidad, convirtiendo su discapacidad en un espacio de lucha constante, de búsquedas de acceder, y dejando a la luz que "El elemento central del concepto de exclusión es la falta de acceso a bienes, recursos o derechos que están disponibles y son accesibles para algunas personas en determinadas situaciones" (Soto, 2003).

RespectoaPolíticas Socialesdel sector, el Mecanismo Nacional de Prevención de la Tortura (MNP), para el cumplimiento de sus funciones, ha definido la política en la cual se encaminará para lograr sus objetivos y específicamente trabaja lo sectorial con un enfoque de prevención o información.

Aunque existen avances, la mayoría de nuestras políticas sociales siguen siendo meramente asistencialistas y entienden a la persona como causante de sus problemas o su situación de vulnerabilidad. Sin embargo es importante rescatar que actualmente existen avances importantes en cuanto a políticas sociales que tienen que ver con género y justamente con discapacidad y prevención de la tortura (Alonso, 2007).

A pesar del gran avance que significa la Convención Internacional por los derechos de las personas con discapacidad y la creación de la SENADIS (Secretaría Nacional por los Derechos Humanos de las 
Personas con Discapacidad) Ley $\mathrm{N}^{\circ}$ 4720/12 en estas áreas de intervención existen varias leyes, normativas vigentes que regulan dichos casos como la Constitución Nacional en varios de sus artículos.

La población de mujeres con discapacidad privadas de su libertad, por el hecho de ser mujeres presentan factores de género que las convierte en potenciales víctimas de vulneración, abandono e incumplimiento de derechos básicos y fundamentales. Siendo la discapacidad un factor que agrava, en un contexto de exclusión dentro de exclusiones (Brogna, 2006).

La discapacidad puede representar un factor agravante de la vulneración y violación de derechos humanos dentro de un penal de mujeres privadas de libertad. El presente estudio se planteó la interrogante ¡Cuáles son las condiciones de salud de mujeres con discapacidad privadas de libertad en un Penal de Mujeres de la ciudad de Asunción, desde un enfoque de derechos?

Viendo la necesidad de visibilizar una realidad que puede encontrarse naturalizada, la vulneración de derechos de personas con discapacidad en un Penal de Mujeres, tomamos una parte de la población para investigar y evidenciar las falencias de nuestro sistema penitenciario en cuanto a protocolos de atención a personas con discapacidad privadas de su libertad.

\section{Objetivo General}

Analizar las condiciones de salud de mujeres con discapacidad privadas de libertad desde la percepción de actores clave de un Penal, en el marco del enfoque de derechos.

\subsection{Objetivos Específicos}

- Describir el marco legal existente respecto a la defensa y protección de derechos de personas con discapacidad en Paraguay.

- Identificar las condiciones de salud de mujeres con discapacidad que se encuentran privadas de libertad en el Penal desde la percepción de actores claves de la institución.

\section{Material y Métodos}

El estudio fue exploratorio con enfoque cualitativo. Fue realizado en el marco del Trabajo Práctico de la Asignatura Investigación Social y Trabajo Social III del Instituto de Trabajo Social de la UNA, con el apoyo del Mecanismo de Prevención contra la Tortura. La técnica utilizada fue de entrevistas en profundidad. Como instrumento se utilizó una guía de preguntas que contenía las categorías de análisis a ser estudiadas.

La muestra estuvo constituida por profesionales de las áreas de interés ubicadas dentro del penal, como ser Trabajo Social, Psicología y Judiciales.

Todas las entrevistas fueron grabadas y transcriptas en medios audiovisuales. Los datos cualitativos se organizaron en categorías de análisis por su significado, por sus cualidades únicas y conocimientos en profundidad. Se procedió a un análisis entre categorías establecidas de antemano, creando una conceptualización por medio de la comparación con otros conjuntos de categorías o grupos.

Se resguardó la identidad de las personas entrevistadas, así como las denuncias $y / o$ situaciones que fueron comentadas durante las entrevistas por mujeres privadas de su libertad, o por funcionarios del penal.

Contamos con los materiales institucionales del MNP (identificación para ingreso autorizado a cualquier área del penal, y materiales informativos del MNP).

\section{Resultados y Discusión}

Luego de las entrevistas y observaciones realizadas, se visualizó la precariedad en la que los profesionales realizan su labor. No cuentan con insumos, capacitación y apoyo necesario para llevar a cabo sus funciones. De acuerdo a lo manifestado por las entrevistadas, 
los organismos ni las autoridades correspondientes cumplen con su rol de velar por el bienestar de las personas con discapacidad privadas de su libertad. Se citaron como ejemplo, que estas áreas no cuentan con un registro de la cantidad de mujeres con discapacidad que se encuentran en el penal actualmente, así como la falta de insumos básicos para llevar a cabo sus funciones. Los mismos tienen que ser adquiridos y gestionados por los profesionales y por sus propios recursos en ocasiones.

Las áreas de Trabajo Social y Psicología de los Institutos penales son las encargadas del manejo de la información y acompañamiento de los casos de mujeres con discapacidad privadas de libertad, de manera a darles un seguimiento y permitir que accedan a sus derechos básicos como ser salud o rehabilitación. Durante las entrevistas realizadas en la Institución, se constató que ninguna de las profesionales de las áreas citadas ha intervenido directamente en casos específicos. Han tomado conocimiento, han escuchado, pero no han trabajado en la planificación de intervenciones para estos casos.

Ninguna de las profesionales entrevistadas al momento de la entrevista tenía conocimiento de lo que establece la ley en cuanto a personas con discapacidad. Han conseguido poco activando con otras instituciones como SENADIS según sus relatos y nunca han recibido capacitación para saber la ruta a seguir en estos casos. Por ende, esa ruta oficial de intervención, no existe.

Finalmente pudimos confirmar que el área de judiciales cuenta con un registro y con fichas de cada una de las internas, donde además de la situación judicial (procesada, condenada), cuentan con informaciones como; estado de salud, observaciones importantes, incidentes que haya tenido dentro del penal, pedido de algunas áreas para el caso, por ejemplo que salga, que acelere su juicio, etc. se visualizó la ausencia de las autoridades competentes, y desesperación y cansancio de las profesionales a cargo. Como bien lo mencionada la entrevistada, "Nosotras también estamos presas".

Los datos facilitados por esta área nos dieron a conocer unos 5 casos de discapacidad dentro del penal, 2 personas con discapacidad física, 1 persona con discapacidad física pero relacionada ya a la tercera edad (74 años), 1 persona con discapacidad intelectual, 1 persona con discapacidad psicosocial.

Además de otras mujeres, que según nos comentan no cuentan con un diagnóstico, pero presentan similitudes o rasgos de discapacidad psicosocial e intelectual.

\section{Conclusiones}

Con los resultados arrojados por las entrevistas realizadas, se puede concluir que, al momento del levantamiento de los datos, el Penal no contaba con un protocolo de atención o ruta de acción para mujeres con discapacidad que son privadas de su libertad. Lo que trae como consecuencia que las mismas vivan en una situación de constante vulneración de derechos y a lo que le sumamos el factor agravante de que no acceden a un servicio de atención o rehabilitación aun cuando lo necesitan por su condición. Esto significa que las mujeres con discapacidad al ingresar al penal quedan totalmente invisibilizadas, no se activa una ruta que prevea que tipo de atención o de tratamiento requiera para acceder a la salud, sus condiciones empeoran por la situación de negligencia o de exclusión de las actividades recreativas o de ocio con que cuentan dentro del penal, y viven su encierro en total abandono.

Por otro lado, aun existiendo un amplio abanico legal y de derechos, que asegura el cumplimiento de ellos y el acceso a sistemas de atención integral hasta la actualidad, en el Penal donde se realizó el estudio, esto es letra muerta cuando son privadas de libertad. Existen esfuerzos de los profesionales del penal del área de 
judiciales y de trabajo social, aunque poco o nada han conseguido. La SENADIS, el ministerio o los mismos jueces, no han dado respuestas a sus peticiones de atención o de evaluación para poder tratar casos, que aunque sean poco (según los registros) representan una situación de violación total de derechos fundamentales y denota la total falta de organización y de inversión en el área, dejando a esta población invisible dentro del penal.

En cuanto a los tipos de discapacidad, prácticamenteporcada tipodediscapacidad hay una mujer privada de libertad (física, psicosocial, intelectual) en el Penal del Buen Pastor y con todas sus implicancias (tipo de tratamiento, necesidades de adecuaciones de accesibilidad, rehabilitación, etc.) los profesionales mismos manifiestas no estar preparados para atender a estos casos, lo que es realmente alarmante pues de no haber capacidad, no deberían de estar en esas condiciones, o bien se deberían de buscar las herramientas para instalar las capacidades adecuadas y asegurar el cumplimiento de los derechos humanos.

$Y$ por último, refiriéndome a la intervención del profesional de trabajo social en la institución, ésta se convierte en una intervención totalmente incompleta e insuficiente. Pues ni siquiera cuenta con insumos, capacidades y apoyo necesario de la institución para sobrellevar los casos. A esto le sumamos que los mismos no han recibido información y posibles rutas de acción para que su intervención pueda ser adecuada al contexto social y de emergencia en que se encuentran las mujeres, y como otro factor agravante la falta de interés hacia los casos de las mujeres con discapacidad, al no contar con registros mínimos de cantidad y tipos que se encuentran hoy, dentro de la institución.

A raíz de todo este contexto registrado en el estudio, se constató la situación de vulneración, las falencias y las realidades de cada profesional dentro del penal. Con miras a convertirla en un primer borrador de puntapié a una tesis que aporte un protocolo de atención a mujeres con discapacidad privadas de libertad.

\section{Referencias Bibliográficas}

Alonso, F. (2007). Algo mas que suprimir barreras: conceptos y argumentos para una accesibilidad universal. Universidad Autonoma de Barcelona. España: Universidad Autonoma de Barcelona .

Bareiro, L. (2003). Compiladora. Discriminaciones y Medidas Antidiscriminatorias. Debate Teórico Paraguayo y Legislación Comparada. Asunción: UNFPA.

Brogna, P. (2006). Un nuevo paradigma de la discapacidad y el rol de los profesionales de la rehabilitación. Buenos Airs, Argentina: El Cisne.

Centro por la Justicia y el Derecho Internacional. (2006). Mujeres Privadas de Libertad. CEJIL, REGIONAL. Brasil: Folio UNO.

Mecanismo Nacional de Prevención de la Tortura de la República del Paraguay. (2014). Informe anual de gestión. Asunción: MNP.

Mecanismo Nacional de Prevención de la Tortura. (2015). El derecho a tener derechos. Realidades del Encierro en el Paraguay. Asunción: MNP.

Moscoso, M. (2011). La discapacidad como diversidad funcional: los límites del paradigma etnocultural como modelo de justicia social. DILEMA.

Paraguay. Ley $\mathrm{N}^{\circ}$ 4720/12, Que crea la Secretaría Nacional por los Derechos Humanos de las Personas con Discapacidad.

SENADIS. (2014). Aproximación a la realidad de las personas con discapacidad. Asuncion: SENADIS.

Soto, C. (Ed.). (2003). Discriminaciónes. Debate teórico paraguayo. Legislación antidiscriminatoria. Asunción. 